\title{
Performance Analysis of Four Wave Mixing: A Non-Linear Effect in Optical Fibers
}

\author{
Devan Bhalla \\ ECE Dept., B.V.C.O.E. \\ New Delhi
}

\author{
Monica Bhutani \\ ECE Dept., B.V.C.O.E. \\ New Delhi
}

\begin{abstract}
Non-Linear effects in Optical fibers are caused due to the refractive index of the optical medium's dependence on the intensity of light. One of the prominent non-linear effects in Optical fibers is the Four-wave mixing (FWM) phenomenon. FWM effect is resonant when the phase matching condition is satisfied. It only occurs for particular combinations of fiber dispersion and signal frequencies. The FWM is a very unpleasant transmission phenomenon occurring in a transparent optical network based on Dense Wave Division Multiplexing, but it could be used advantageously for implementing optical devices such as wavelength converters, parametric amplifiers, optical de-multiplexers, chromatic dispersion compensators, as well as signal to noise regenerators. This paper discusses the effect of channel spacing, laser power, and dispersion, length of the optical fiber and the variation of input power to compensate the effect of FWM when implemented in a short haul environment.
\end{abstract}

\section{General Terms}

Optical Fiber Non-Linearity, Four Wave mixing

\section{Keywords}

Dispersion, Bit rate, Channel Spacing, Inter-channel Crosstalk, Laser Power

\section{INTRODUCTION}

When a high-power optical signal is launched into an optical fiber, the linearity of the optical response is lost due to the dependence of the medium's refractive index on the intensity of light in the medium. One such nonlinear effect, which is due to the third-order electric susceptibility, is called the optical Kerr effect. Four-wave mixing (FWM) is a type of optical Kerr effect [1], and occurs when light of two or more different wavelengths is launched into an optical fiber. Generally speaking FWM occurs when light of three different wavelengths is launched into a fiber, giving rise to a new wave, the wavelength of which does not coincide with any of the original signal wavelengths. Moreover, FWM is a kind of an optical parametric process under which the optical fiber serves a passive role of a medium for providing interaction among the optical waves and involves the modulation of the refractive index [8]. The origin of parametric processes lies in the non-linear response of bound electrons of a material to an applied optical field. More specifically, the polarization induced in the medium is not linear in the applied field but contains nonlinear terms, whose magnitude is governed by the nonlinear susceptibilities of different order particularly the third order susceptibility $\chi(3)$ of the medium[7] given by-

$\mathrm{P}_{\mathrm{NL}}(\mathrm{r}, \mathrm{t})=\epsilon_{\theta} \mathrm{X}^{(3)} \mathrm{E}^{(3)}(\mathrm{r}, \mathrm{t})$

-Eq.(1.1).
In general FWM involve nonlinear interaction among four optical waves and is referred to as third order parametric process because it is caused by the third order nonlinear susceptibility.

The nonlinear effects degrade the system performance like by introducing interference, distortion excess attenuation of the optical signals. The nonlinear effects tend to manifest themselves when optical power is very high, thereby gaining importance in DWDM. If in a WDM system the channels are equally spaced, the new waves generated by FWM will fall at the original channel frequencies and, thus, will give rise to crosstalk. FWM needs to be minimized for efficient transfer of information on the Optical Fiber Link. In the transmission of dense wavelength-division multiplexed (DWDM) signals [2], FWM is to be avoided, but for certain applications, it provides an effective technological basis for fiber-optic devices. FWM also provides the basic technology for measuring the nonlinearity and chromatic dispersion of optical fibers.

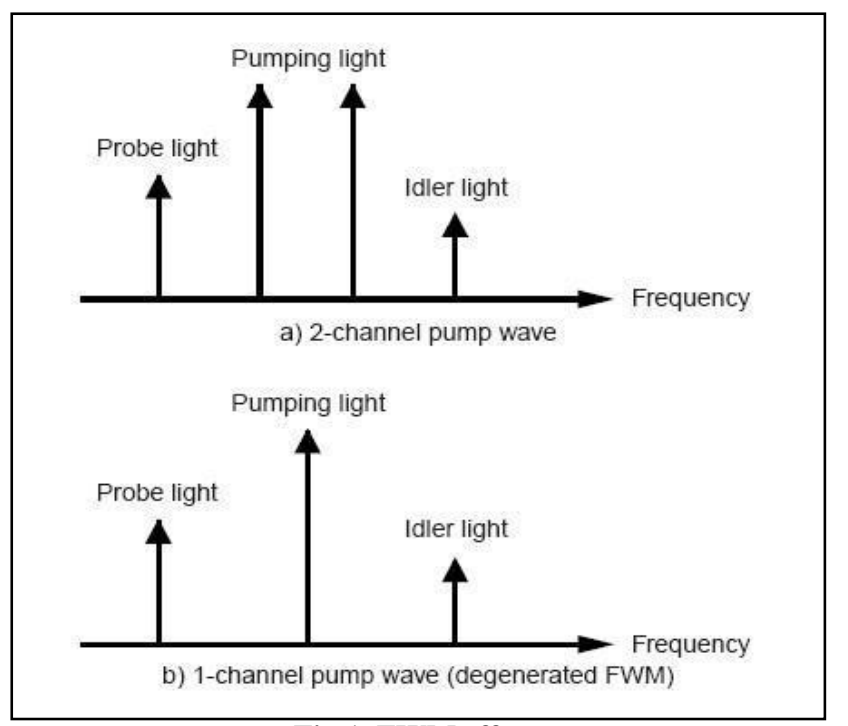

Fig.1. FWM effect

Fig.1. [5] is a schematic diagram that shows four-wave mixing in the frequency domain. As can be seen, the light that was there from before launching, sandwiching the two pumping waves in the frequency domain is called the signal/probe light whereas the idler frequency is the $f_{\text {idler. }}$ which is represented as follows-

$f_{\text {idler }}=f \mathrm{p} 1+f \mathrm{p} 2-f$ probe

-Eq.(1.2).

where $f_{p 1}$ and $f_{p 2}$ are the pumping light frequencies, and $f_{\text {probe }}$ is the frequency of the probe light. This condition is called 
the frequency phase-matching condition [6].

There are different types of FWM depending on the involved optical waves. In the case of three distinct optical wave frequencies three photons transfer their energy to a single photon at the frequency-

$\omega 4=\omega 1+\omega 2+\omega 3$

This is so called non-degenerate FWM [3]. For this process it is difficult to satisfy phase matching in OF with high efficiency. Another case is when there are two optical wavelengths at distinct frequencies $\omega 1$ and $\omega 2$. Then photons from frequencies $\omega 1$ and $\omega 2$ are annihilated with simultaneous creation of two photons at frequencies $\omega 3$ and $\omega 4$ such that-

$\omega 3+\omega 4=\omega 1+\omega 2$

-Eq.(1.4).

this is the partially degenerate case [3]. The phase-matching requirement for this process to occur is given by-

$k=k 3+k 4-k 1-k 2=0$

-Eq.(1.5)

\section{SIMULATION SETUP}

The continuous wave laser (L1-L10) in Fig.2. was used to create the carrier signal. In this setup, ten users were taken in account whose wavelengths have a specific difference i.e. channel spacing between them. The wavelengths of following users were set as per the spacing requirement i.e. at wavelength difference of $6.25 \mathrm{GHz}, 10 \mathrm{GHz}, 20 \mathrm{GHz}, 25$ $\mathrm{GHz}, 75 \mathrm{GHz}$. The data source (ds1-ds10) was used to generate the random input data bit sequence at the rates of 3,5 and $10 \mathrm{Gbps}$. (f1) of length $0.8 \mathrm{~km}$. All the attenuation, dispersion and non linear effects were activated. The in-line amplifier (a2) amplifies the signal in the transmission medium itself. Then the signal was again passed through a fiber (f2) of length 0.8 $\mathrm{Km}$. Then pre-amplifier (a3) was used to amplify the signal before it was allowed to enter into the receiver section. After amplification, the signal reached the receiver where it was demultiplexed by using a splitter (s1) which split the signal into the same number of signals as were transmitted. The photodiode (p1) was used for optical to electrical conversion. Then the signal was passed through the Raised Cosine filter (Lf1) which was made to work as low pass filters and the final output signal was received. An optical scope (probe1) was attached at the output of combiner to examine the input signal. Another optical scope (probe2) was placed at the output of splitter to examine the four wave mixing effect in frequency spectrum. An electrical scope was kept at the receiver output to examine the eye diagram, BER, Q-factor.

Parametric values used for study are as follows-

\begin{tabular}{|c|c|}
\hline Parameter & Value \\
\hline Optical fiber length & $0.8 \mathrm{~km}$ \\
\hline Frequency operational range & $190-194 \mathrm{THz}$. \\
\hline Input channels used & 10 \\
\hline Bit rate & $10 \mathrm{Gbps}$ \\
\hline
\end{tabular}

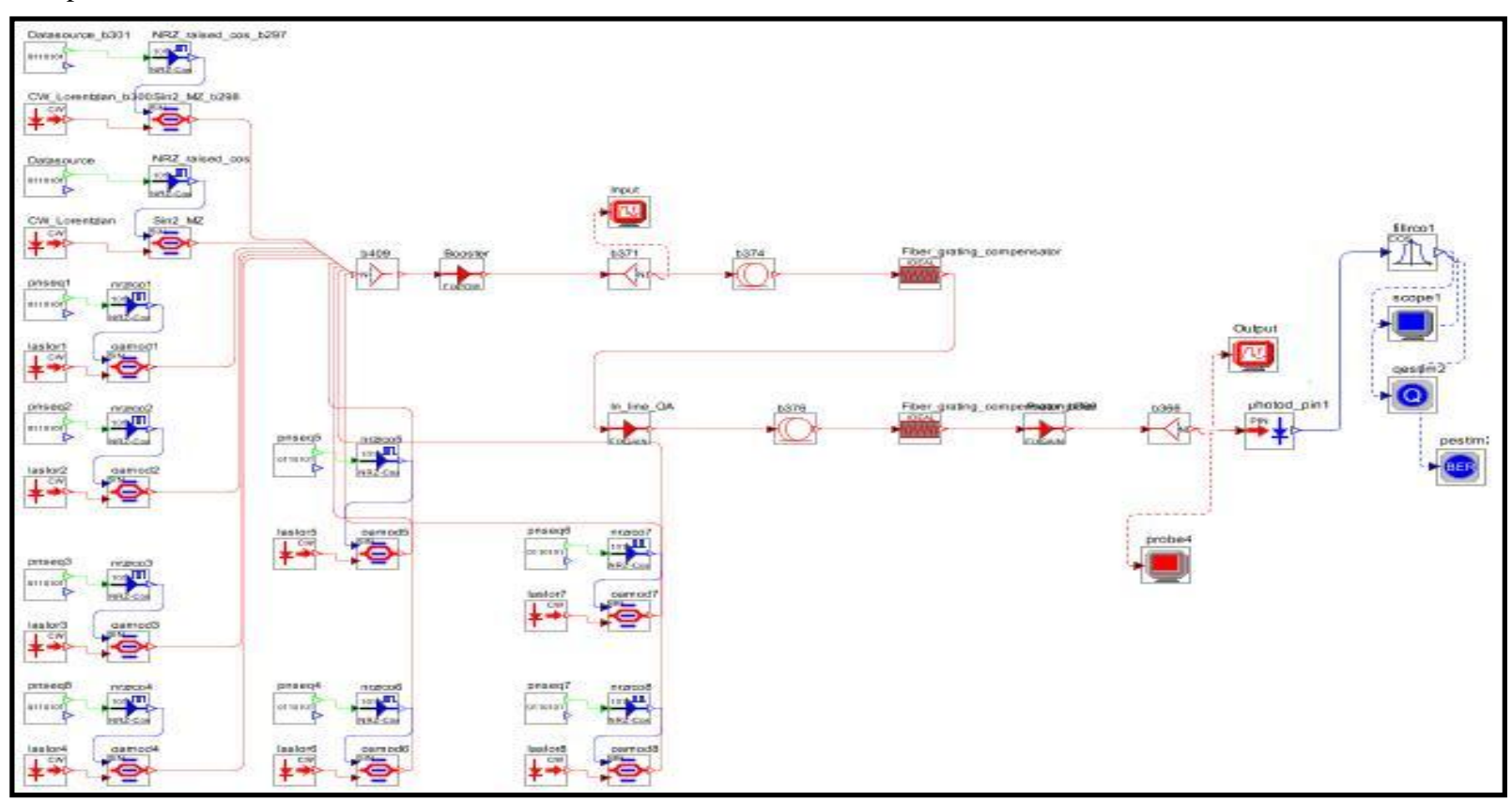

Fig.2. Simulation Setup

The light signal modulated the input data. The modulator $(\mathrm{m} 1-$ m8) was driven by the modulator driver (d1-d10) which decides the input data format. The input data format used here is NRZ raised cosine. The modulated data from all the users is combined using a combiner (c1). The post amplifier (a1) amplifies the signal before being allowed to enter into the fiber to avoid losses. Then this signal was sent over the fiber 


\section{SIMULATION RESULTS AND}

\section{ANALYSIS}

\subsection{Two Channel Setup}

As shown in Fig.3.(a) two signal channels at the input are used to explain the basic FWM effect taking place within the Optical Fiber. Here the frequency used is $193 \mathrm{THz}$.

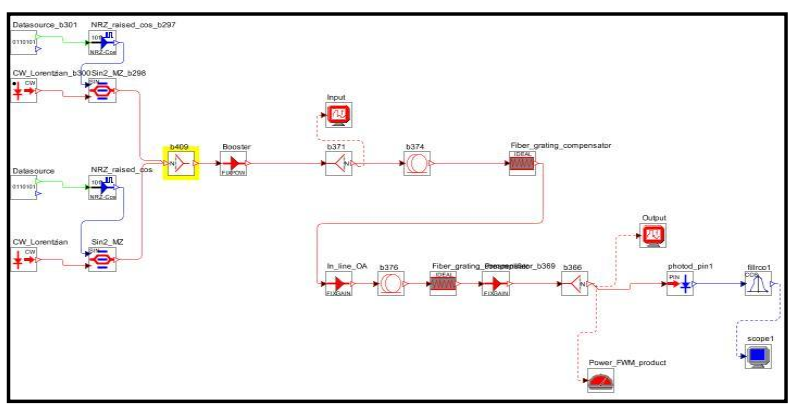

Fig.3.(a). Simulation Setup

The two frequencies are of $193.025 \mathrm{THz}$. and $193.075 \mathrm{THz}$ as shown in Fig.3.(b).

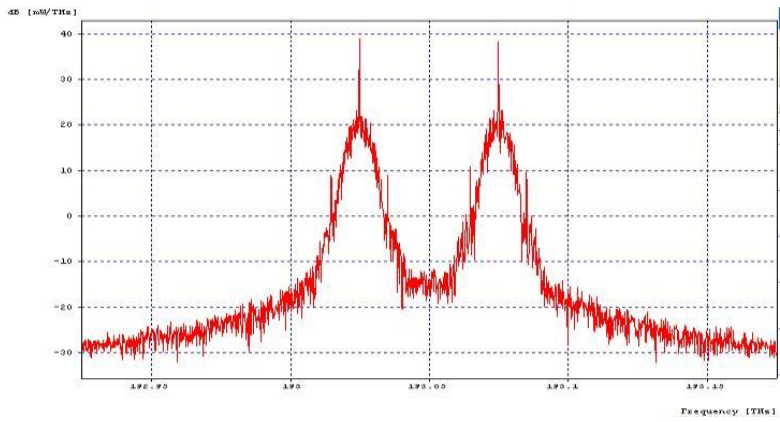

Fig.3.(b). Input

The output frequencies as shown in Fig.3.(c). are: $\mathrm{F} 1=192.975 \mathrm{THz}, \mathrm{F} 2=193.025 \mathrm{THz}, \mathrm{F} 3=193.075 \mathrm{THz}$ and F4=193.125 THz. These output frequencies have been obtained as per the rule of:

$\mathrm{F}_{\mathrm{abc}}=\mathrm{f}_{\mathrm{a}}+\mathrm{f}_{\mathrm{b}}-\mathrm{f}_{\mathrm{c}}$

-Eq.(3.1.).

Where $f_{a}, f_{b}$ and $f_{c}$ are the incident frequencies at the input of the optical fiber and there can be a number of such combinations.

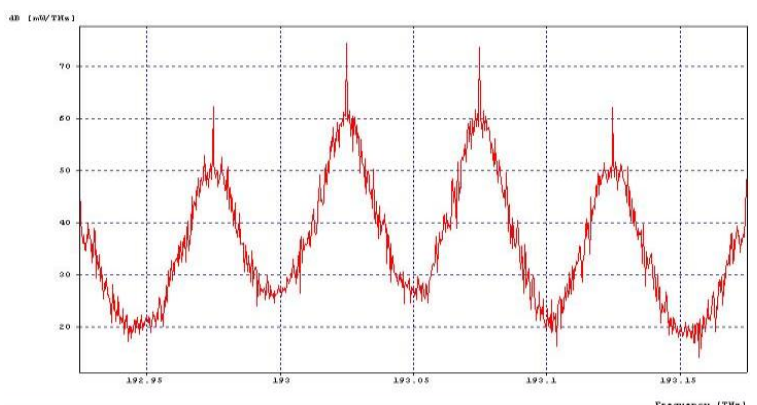

Fig.3.(c). Output

\subsection{Effect on FWM due to Dispersion}

Dispersion is the phenomenon of the spreading of the signal in the time and the frequency domain and as such leads to the spreading of the signal. Four Wave Mixing effect varies inversely with the Dispersion and this has been strengthened by this result.

Here, D is varied from 2 to 50 while keeping the length of the Optical Fiber constant at $0.8 \mathrm{Km}$. The Parametric Run feature of OptSim 5.2 was used for this implementation with the following Dispersion values-

\begin{tabular}{l|l}
\hline Run \# & Param 1 \\
\hline Parameter & dispersion \\
\hline Run 1 & 2.0 \\
\hline Run 2 & 5.0 \\
\hline Run 3 & 7.0 \\
\hline Run 4 & 10.0 \\
\hline Run 5 & 15.0 \\
\hline Run 6 & 20.0 \\
\hline Run 7 & 35.0 \\
\hline Run 8 & 50.0
\end{tabular}

Fig.3.(d). Parametric Scan on Dispersion in $\mathrm{ps} / \mathrm{nm} / \mathrm{km}$

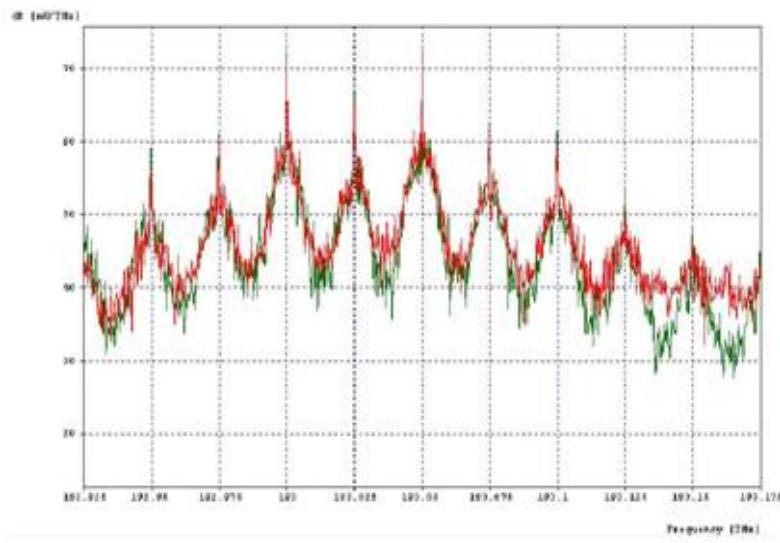

Fig.3.(e). Output Optical Spectrum with Parametric Scan on Dispersion

Here, the portion in Red indicates value at lower value of Dispersion and those in Green indicate final value when Dispersion was increased.

\subsection{Effect on FWM due to Length of Optical fiber link}

The parametric scan applied is on length (L) of the Optical fiber link through which the signal is being propagated. As the Length of the fiber increases, so does the FWM effect as it being a non-linear effect gets accumulated according to the length of the medium.

\begin{tabular}{|l|l}
\hline \multicolumn{1}{|l}{ Run \# } & \multicolumn{1}{c}{ Param 1 } \\
\hline Parameter & \multicolumn{1}{c}{ L } \\
\hline Run 1 & 0.1 \\
\hline Run 2 & 0.5 \\
\hline Run 3 & 0.8 \\
\hline Run 4 & 1 \\
\hline Run 5 & 5 \\
\hline Run 6 & 10 \\
\hline Run 7 & 20 \\
\hline Run 8 & 50 \\
\hline Run 9 & 75 \\
\hline Run 10 & 100 \\
\hline
\end{tabular}

Fig.3.(f). Parametric Scan on Length in km 
Here the Parametric Scan was applied on the Length and the Output Optical Spectrum confirmed that the non-linearity was increasing in proportion with the length of the optical fiber.

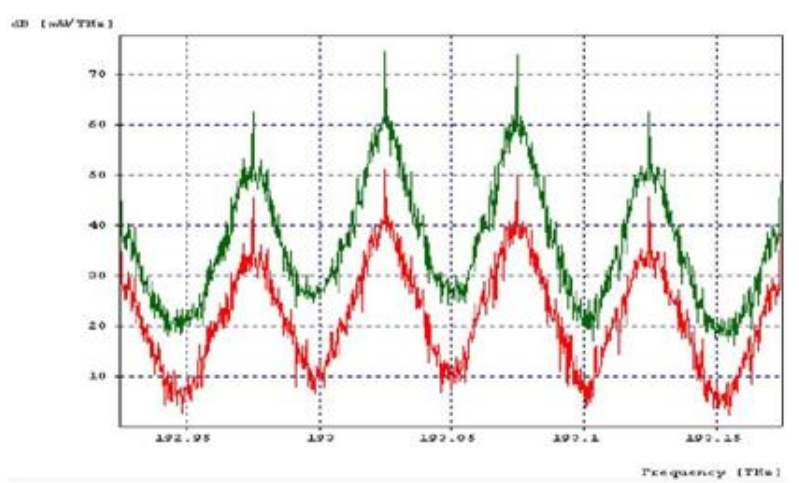

Fig.3.(g). Output Optical Spectrum with Parametric Scan on Length

\subsection{Channel Spacing Effect on FWM}

As the channel spacing increases the FWM effect gets minimized as it increases the group velocity mismatch between the channels [9]. This feature of providing higher channel spacing needs to be employed for long distance communication using the Optical Fiber links. The channel spacing was increased from $6.25 \mathrm{Gbps}$ up till $25 \mathrm{Gbps}$ to study the effects in the Output Optical Spectrum.

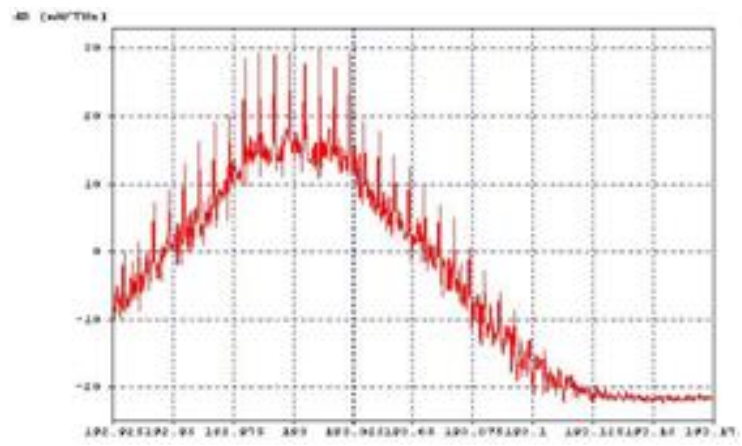

Fig.3.(h). Output Optical Spectrum with Channel Spacing of 6.25 Gbps

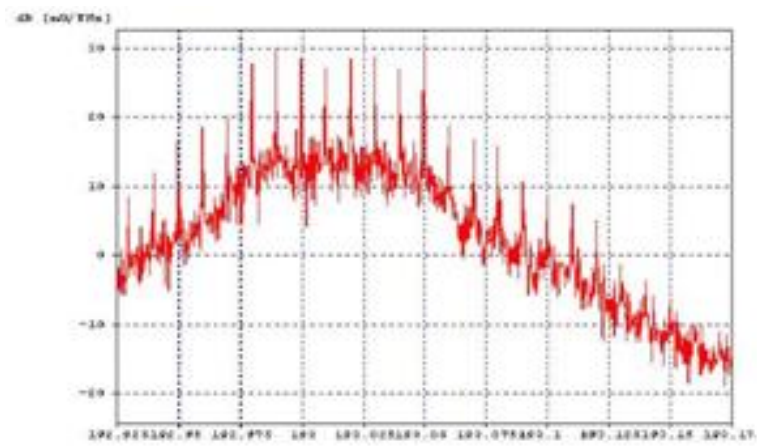

Fig.3.(i). Output Optical Spectrum with Channel Spacing of 10 Gbps

Channel Spacing when adequately provided reduces interference among the neighboring signal peaks and helps in avoiding interference and thus reduces loss of information and counters distortion effectively.

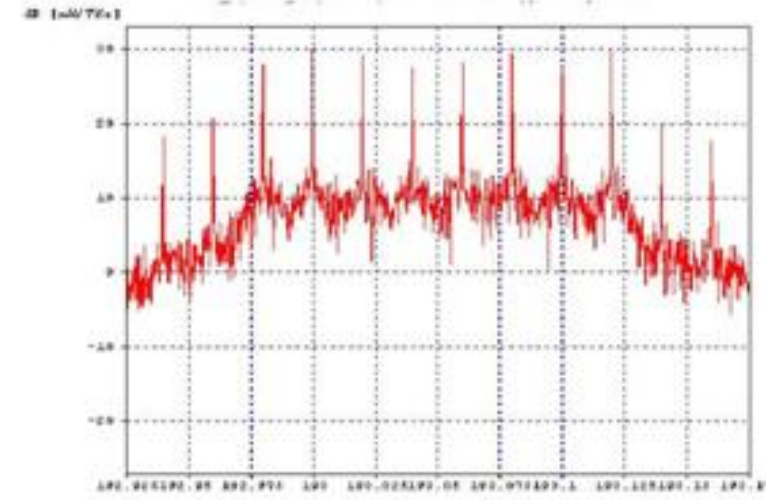

Fig.3.(j). Output Optical Spectrum with Channel Spacing of 20 Gbps

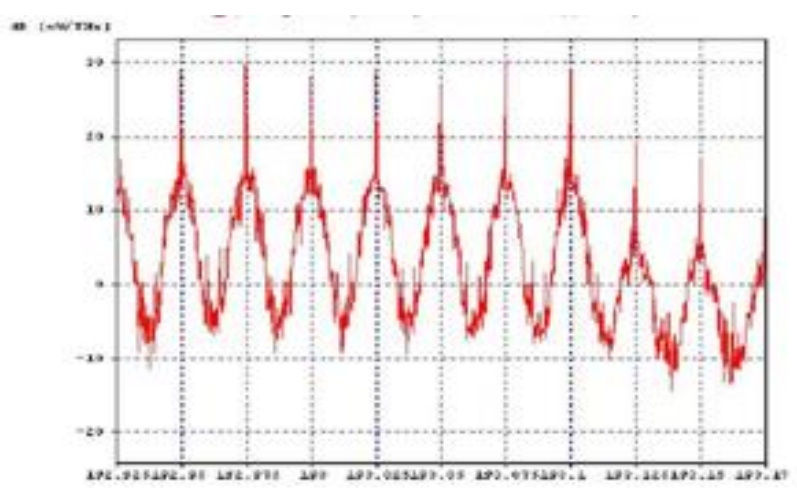

Fig.3.(k). Output Optical Spectrum with Channel Spacing of 25 Gbps

Here, it was noted that as the channel spacing increased, the output optical spectrum's clarity increased and the performance of the system improved.

On increasing the channel spacing, the interference between input frequencies decreases and hence, the four wave mixing effect also decreases. Moreover, on increasing the number of input channels/users, the interference again increases and thus, the four wave mixing effect also increases which was deduced from the following obtained Eye Diagrams as shown in Fig.3.(1). and Fig.3.(m).

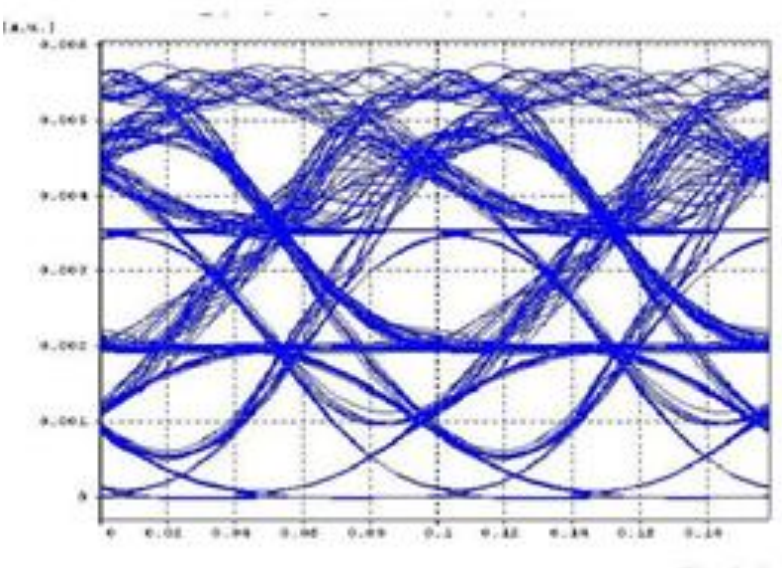

Fig.3.(1). Eye Diagram with a Channel Spacing of 25 Gbps in a Two Channel System 


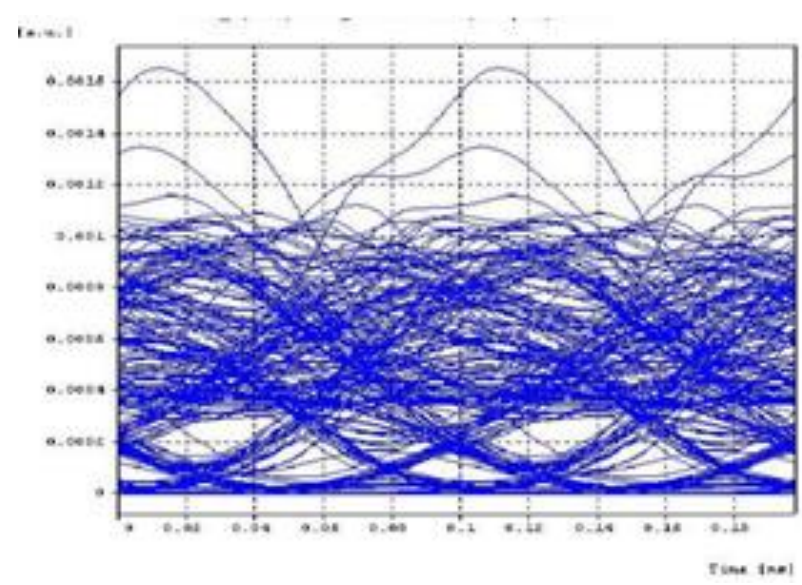

Fig.3.(m). Eye Diagram with a Channel Spacing of 25 Gbps in a Four Channel System

The above eye diagrams show that as the number of input channels increases, the clarity of eye diagram diminishes.

\subsection{Effect of Laser Power on FWM}

The Laser is used as a source in an optical communication system just like a carrier signal. As the power of the involved laser increases the non-linear effect of FWM decreases.

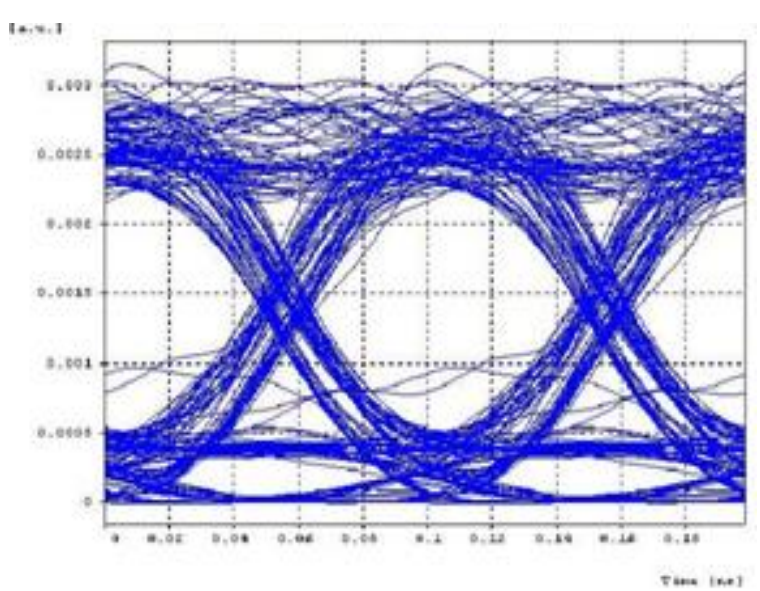

Fig.3.(n). Eye Diagram with Power level of $-5 \mathrm{~dB}$

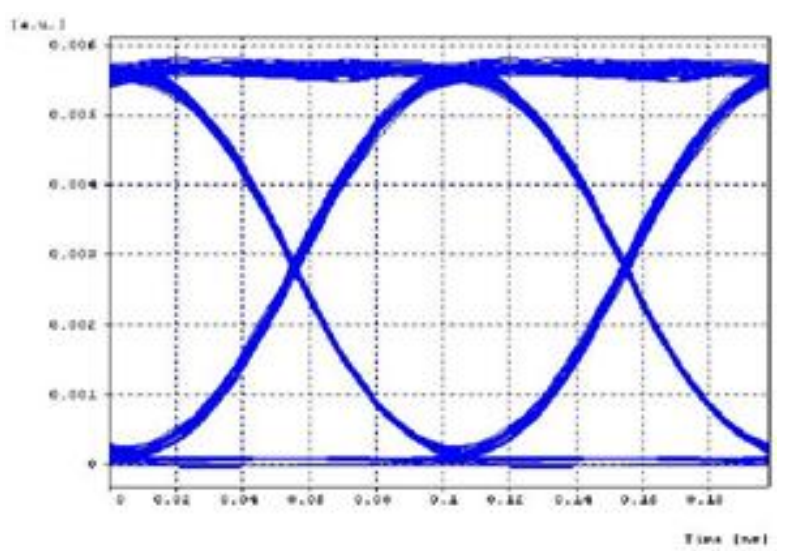

Fig.3.(o). Eye Diagram with Power level of $5 \mathrm{~dB}$
The Laser Power was varied from $-5 \mathrm{~dB}$ to $5 \mathrm{~dB}$ and it was deduced that as the power level increases the FWM effect decreases and the clarity of the eye diagram increases.

\subsection{Effect of Bit Rate on FWM}

The Four Wave Mixing effect is not affected by bit rate unlike that in SPM or XPM. [4]

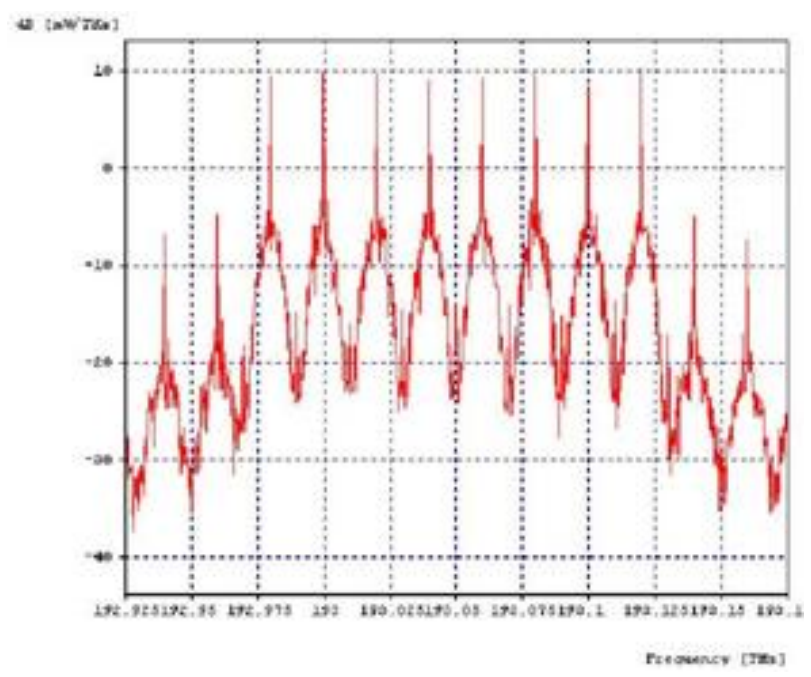

Fig.3.(p). Eye Diagram with Bit rate of 10 Gbps

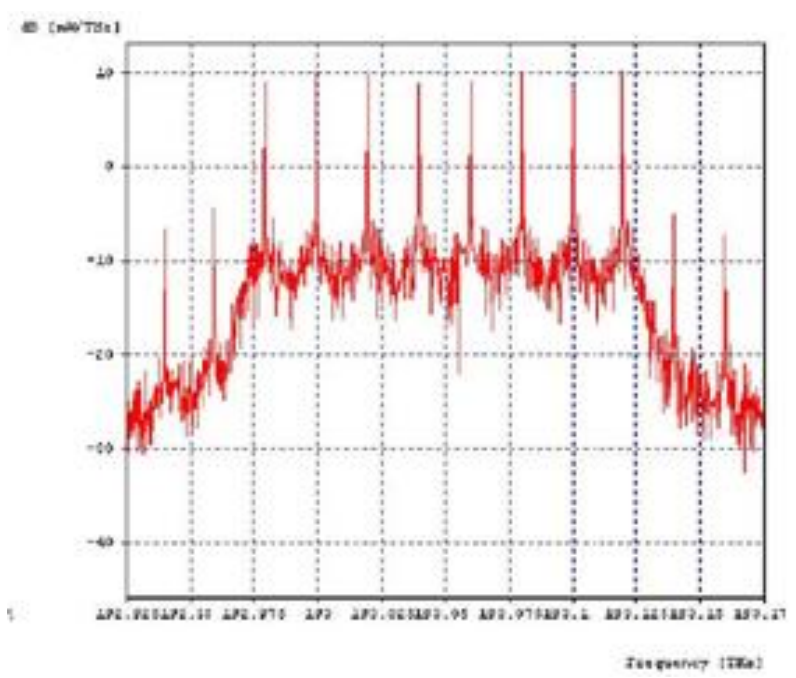

Fig.3.(q). Eye Diagram with Bit rate of $20 \mathrm{Gbps}$

There is no change in the output on changing the source's data rates in terms of sidebands of the FWM effect but the attenuation in terms of power increases as the data rate is increased.

\section{CONCLUSIONS AND FUTURE SCOPE}

In this paper the Four Wave Mixing effect has been analyzed and techniques through which it can be effectively reduced over the short haul communication system have been suggested. Here, it has been demonstrated that the effect of increasing the channel spacing effectively reduces this nonlinear effect which can be effectively adopted when the number of channels increases for sending information signals over the optical link. FWM has been shown to be independent of bit-rate unlike the other non-linear effects of XPM and SPM. Also, FWM gets accumulated with the increase in the 
optical fiber link's length and continues accumulating as the length increases thus making it of critical importance to compensate it and improve the signal quality. The effect of dispersion has also been highlighted with it being inversely related to this non-linear optical Kerr effect. Also, the results show that the FWM effect is dependent on the input optical signal power. The further analysis will aim at comparing different optical fibers in a short haul as well as a long haul environment. The focus will be on to include different components like VCSEL laser, varying the filters, modulators and the signal drivers to be used to contrast performance and reach at an optimum optical communication system.

\section{REFERENCES}

[1] Liang Wang, Shu C, IEEE 2013, Enhanced performance of four wave mixing wavelength conversion through dynamic control of optical phase

[2] Selvamani, IEEE 2011, Suppression of four wave mixing by optical phase conjugation in DWDM fiber optic link

[3] Supe A., Porins J., 2011, Comparison of different FWM Realization methods in Optical Fibre.
[4] Ezra M.Ip, Joseph M.Kahn, Journal of Lightwave Technology, IEEE 2010, Fiber impairment compensation using coherent detection and digital signal processing.

[5] Osamu Aso, Masateru Tadakuma, Shu Namiki, Four Wave mixing in Optical Fibre and its Applications, WP team, Opto Technology Lab., R\&D Div.

[6] Sabapathi T., Sundaravadivelu S., Elseiver Optik 2010, Analysis of bottlenecks in DWDM fiber optic communication system.

[7] Toulose J., Journal of Lightwave Technology IEEE 2005, Optical Nonlinearities in Fibers: Review, Recent Examples, and Systems Applications.

[8] Agrawal GP, 2001, Nonlinear Fiber Optics, $3^{\text {rd }}$ Ed., Academic Press, San Diego, CA.

[9] Forghieri F, Tkach RW, Carptyvy, Marcause D, IEEE Photonics Tech. Lett. 6:6, 1994, Reduction of four-wave mixing crosstalk in WDM systems using unequally spaced channels. 